\title{
Horizons philosophiques
}

Savoirs traditionnels, savoirs modernes et enseignement

philosophique de niveau collégial dans la modernité québécoise, Louise LeVasseur, thèse de doctorat présentée à l’Université de Montréal en 1997.

\section{Maurice Burgevin}

Volume 11, numéro 1, automne 2000

L’amodernité de la photographie?

URI : https://id.erudit.org/iderudit/802958ar

DOI : https://doi.org/10.7202/802958ar

Aller au sommaire du numéro

Éditeur(s)

Collège Édouard-Montpetit

ISSN

1181-9227 (imprimé)

1920-2954 (numérique)

Découvrir la revue

Citer ce compte rendu

Burgevin, M. (2000). Compte rendu de [Savoirs traditionnels, savoirs modernes et enseignement philosophique de niveau collégial dans la modernité québécoise, Louise LeVasseur, thèse de doctorat présentée à l'Université de Montréal en

1997.] Horizons philosophiques, 11(1), 153-155. https://doi.org/10.7202/802958ar d'utilisation que vous pouvez consulter en ligne.

https://apropos.erudit.org/fr/usagers/politique-dutilisation/ 


\section{Savoirs traditionnels, savoirs modernes et enseignement philosophique de niveau collégial dans la modernité québécoise, Louis LeVasseur, thèse de doctorat présentée à l'université de Montréal en 1997.}

Le numéro précédent de cette revue publiait un compte-rendu de lecture de l'ouvrage de Ferry et Renaud, Philosopher à 18 ans, qui dressait un bilan de l'enseignement de la philosophie en France au niveau pré-universitaire. Un travail du même ordre a été fait au Québec dans une thèse de doctorat de Louis LeVasseur, présentée à l'université de Montreal en 1997 : Savoirs traditionnels, savoirs modemes et enseignement philosophique de niveau collégial dans la modemité québécoise. Cette thèse est la principale source d'informations sur l'enseignement de la philosophie au collégial utilisée dans l'ouvrage écrit sous la direction de Raymond Klibansky et de Josiane Boulad-Ayoub et publié aux Presses de l'Université Laval en 1998 : La Pensée philosophique d'expression française au Canada.

LeVasseur, selon ses propres termes, étudie la dérive des savoirs, de Platon à Kant et à Lyotard. L'étude de l'enseignement de la philosophie dans les collèges publics du Québec ("cégeps"), tel qu'il apparaît essentiellement dans les textes officiels et les plans d'études des professeurs, lui semble illustrer la conception actuelle du savoir. II propose, au sujet de l'enseignement collégial de la philosophie, une synthèse remarquable et une critique qui peut intéresser ou agacer, mais qui, en tout cas, ne peut pas laisser indifférent.

II divise l'histoire de l'enseignement de la philosophie dans les collèges publics en trois périodes : les années 1967-1977, les années 1977-1984, et les années 1984-1995. Les années 1977 à 1984 sont présentées comme des années de transition; nous nous contenterons donc ici de comparer les premières et les demières années.

Durant les années 1967-1977, la distanciation et l'appropriation sont les axes de l'enseignement de la philosophie. La distanciation est un moyen d'arriver à l'appropriation. La distanciation peut être vue sous divers angles : une prise de conscience des contradictions du vécu, une prise de conscience de l'oppression du capitalisme ou simplement une critique du milieu socioculturel. L'appropriation, quant à elle, peut être une démarche autonome de reformulation d'un système de valeurs personnel, une recherche d'un sens à sa vie ou bien une prise de conscience de la nécessité d'une révolution et éventuellement une décision de s'y engager - un professeur aurait, par exemple, indiqué dans son plan d'études qu'il était souhaitable que ses élèves aillent manifester devant une usine de boissons gazeuses 7up.

Face au Rapport Nadeau, publié en 1975, qui remettait en question la pertinence d'un enseignement de la philosophie (l'apprentissage des habiletés intellectuelles qu'on y faisait pouvant être fait ailleurs), Jacques Ouellet, alors coordonnateur provincial, a immédiatement répliqué que l'enseignement de philosophie ne devait pas être évalué selon des critères utilitaristes:

Pour l'étudiant (...) dont la vie ne s'arrête ni au rôle, ni à la fonction spécifique de sa profession, la philosophie offre l'occasion de réfléchir méthodiquement sur les raisons de la vie, de l'être et des choses, de poser les questions fondamentales, de se situer dans le monde et la société; d'apprendre à justifier ses préférences et ses valeurs d'une manière critique et systématique. (cité p. 289) 
Dans les années 1984-1995, l'accent est de plus en plus mis sur l'histoire de la philosophie. La philosophie est devenue un cours d'autodéfense intellectuelle, selon une expression de l'époque, dans lequel on s'arme de logique, d'épistémologie et d'histoire de la philosophie. II importe d'examiner sans prévention les divers systèmes philosophiques et de montrer qu'ils sont relatifs à un contexte socio-historique. On peut donc (on doit donc?) être ouvert à tous les systèmes philosophiques.

On favorise la distanciation; mais il est de plus en plus difficile à l'étudiant de se départir de sa distance et de s'engager ou de s'investir dans une prise de position personnelle, à moins que cette position ne soit le relativisme.

Cependant, vers la fin des années 80 , les critiques dirigées contre la philosophie réapparaissent. Celle-ci est sommée de montrer son utilité pour la nouvelle économie. On décide alors de mettre l'accent sur le développement des habiletés intellectuelles : tout travailleur devra savoir analyser, synthétiser, problématiser pour produire efficacement. En 1992, selon le coordonnateur de l'époque, Cohen Bacrie:

\begin{abstract}
S'il s'agit d'enseigner la philosophie pour les idées qu'elle met en jeu, ce qui contribue à l'élargissement de la conscience, il faut reconnaître que l'effet recherché par cet enseignement est de pratiquer et de développer des capacités intellectuelles et langagières de niveau supériour. II faut reconnaîre que ces compétences sont adéquates aux nouveaux besoins. (cité p. 366 )
\end{abstract}

Si on compare le texte de Jacques Ouellet avec celui de Cohen-Bacrie, on peut mesurer le chemin fait entre 1975 et 1992. Au lieu de former des travailleurs contestataires comme dans les années 70, la philosophie formera donc des travailleurs compétents, à l'esprit ouvert.

En bref, dans les années 70, l'étudiant est interpellé, la philosophie a des perspectives normatives (éthiques et politiques) : elle est conçue comme une critique du milieu socioculturel. Dans les années 90 , le centre de l'enseignement n'est plus l'étudiant, mais la philosophie elle-même : le cours vise l'érudition, il veut initier l'étudiant à des systèmes philosophiques et lui apprendre à s'en distancier en montrant leur relativité. En outre, dans les années 70 , le contexte socioculturel marque notre enracinement et la philosophie le conteste; dans les années 90 , le contexte socioculturel montre la relativité de toutes les doctrines.

La conclusion de LeVasseur n'est pas plaisante pour les professeurs de philosophie. Selon lui, suite à l'effondrement du thomisme, la société québécoise a cherché à se trouver d'autres principes unificateurs : les professeurs de philosophie ne voulaient pas se mettre au service de la classe dominante; le pouvoir, de son cóté, ne pouvait supporter que ces principes soient inspirés du marxisme. On a donc fait un compromis, on a renoncé à tenter de trouver des principes communs et on s'est engagé dans un pluralisme relativiste. Mais en choisissant d'être académique, historique et relativiste, postmoderne, l'enseignement de la philosophie ne favorise plus l'engagement de l'étudiant dans la culture et la vie sociale. Notre enseignement a donc (excusez l'impudence de LeVasseur), un résultat semblable à l'enseignement de la philosophie thomiste!

Le genre littéraire "note de lecture» exige que l'auteur fasse part de ses jugements personnels. Qu'est-ce qu'il serait possible et souhaitable d'ajouter? II serait bien tentant de dire 
que l'ouvrage parle de lui-même, surtout lorsqu'on enseigne soi-même la philosophie et qu'on est d'accord avec LeVasseur. Cette étude met, en effet, le doigt sur nos contradictions : nous sommes des serviteurs de l'État, même si nous affirmons bien haut dans nos premiers cours notre allégeance à Socrate. Nous sommes des "Socrate fonctionnaires", a-t-on déjà écrit. Cette étiquette est agaçante. Nous sommes pris avec les impératif́s de l'État relayés par les exigences de nos patrons et les attentes de nos étudiants.

Plusieurs de nos patrons ont été nos élèves dans les années 70. Ils n'ont pas toujours été édifiés par le sérieux de notre enseignement, à cette époque marquée par Refus Global. Nous devons donc leur donner des garanties. Nous sommes devenus académiques, nous donnons un contenu précis à notre enseignement, nous enseignons l'histoire de la philosophie, même si cette histoire n'a souvent aucun enracinement dans la culture des étudiants, dans la culture nord-américaine, ni, peut-être même, dans la culture occidentale de l'an 2000. À l'ère de l'ouverture sur le monde et du triomphe de la technique, il est difficile de faire partager notre enthousiasme pour le "miracle grec".

De plus, pour mériter notre salaire, nous avons une obligation de résultat, nos patrons attendent de nous un rendement quantifié. En conséquence, même si nous n'arrêtons pas de nous plaindre du faible niveau de nos étudiants (tout comme nos collègues de France), nous devons découvrir, à la fin de la session, que, tout compte fait, la plupart d'entre eux méritent au moins $60 \%$.

De leur cóté, nos étudiants, en arrivant au niveau collégial, ont hâte de se spécialiser. Ils tolèrent les cours obligatoires de «français" parce que le français, ca sert. Quant à l'éducation physique, c'est plaisant. Mais la philosophie et la culture générale, c'est tout simplement "platte". Nous devons donc trouver un moyen de retenir nos étudiants dans nos classes. Des évaluations gratifiantes, cela ne peut pas nuire.

Notre maître, Socrate, n'acceptait aucun compromis; sa condamnation à mort a été la conséquence de son entêtement. Si nous, les professeurs de philosophie de collèges, étions aussi purs que Socrate, on ne nous condamnerait pas à mort (cette peine n'existe plus sur nos terres), on nous condamnerait au chômage. Mais, après avoir fait de Socrate un si bel éloge dans nos premiers cours, et souvent sans même marquer l'hiatus, nous enseignons la relativité de toute opinion, comme Protagoras, et nous formons d'habiles discoureurs compétents pour démontrer n'importe quoi.

Inconséquences? Paradoxes? Contradictions? Certainement. On pourrait pourtant donner un enseignement à la fois plus cohérent et plus signifiant. Pourquoi, par exemple, ne pas essentiellement s'interroger sur les conséquences de la mort de Dieu, comme le suggère Luc Ferry? (Ferry doit avoir un sens de ce qui est admissible pour un État puisqu'il est lui-même chargé de faire à l'État français des recommandations sur tous les programmes du secondaire.) Malgré toutes ces critiques, en toute bonne foi, l'auteur de ces lignes n'a pas encore honte de recevoir un salaire de "prof. de philo.", il parie encore que son travail reste une aide signifiante pour la vie personnelle, culturelle et sociale de ses étudiants. Misères de l'enseignement de la philosophie, certes; mais cet enseignement, qu'on pourrait développer ailleurs, a aussi ses grandeurs.

Maurice Burgevin

Cégep de Saint-Hyacinthe 